\title{
Stargardt macular dystrophy and evolving therapies
}

Rehan M. Hussain, Thomas A. Ciulla, Audina M. Berrocal, Ninel Z. Gregori, Harry W. Flynn, Byron L. Lam

\begin{abstract}
$\underline{\text { Abstract }}$
Introduction: Stargardt macular dystrophy (STGD1) is a hereditary retinal degeneration that lacks effective treatment options. Gene therapy, stem cell therapy, and pharmacotherapy with visual cycle modulators (VCMs) and complement inhibitors are discussed as potential treatments.
\end{abstract}

Areas Covered: Investigational therapies for STGD1 aim to reduce toxic bisretinoids and lipofuscin in the retina and retinal pigment epithelium (RPE). These agents include C20D3-vitamin A (ALK-001), isotretinoin, VM200, emixustat, and A1120. Avacincaptad pegol is a C5 complement inhibitor that may reduce inflammation-related RPE damage. Animal models of STGD1 show promising data for these treatments, though proof of efficacy in humans is lacking. Fenretinide and emixustat are VCMs for dry AMD and STGD1 that failed to halt geographic atrophy progression or improve vision in trials for AMD. A1120 prevents retinol transport into RPE and may spare side effects typically seen with VCMs (nyctalopia and chromatopsia). Stem cell transplantation suggests potential biologic plausibility in a phase I/II trial. Gene therapy aims to augment the mutated $A B C A 4$ gene, though results of a phase I/II trial are pending. 
Expert Opinion: Stem cell transplantation, $A B C A 4$ gene therapy, and VCMs offer biologically plausible treatment mechanisms for treatment of STGD1. Further trials are warranted to assess efficacy and safety in humans.

Keywords: C20-D3-vitamin A, Stargardt macular dystrophy, visual cycle, complement inhibition, stem cell therapy, $A B C A 4$, gene therapy, SAR422459

\section{Introduction}

Autosomal recessive Stargardt macular dystrophy (STGD1) is a retinal dystrophy resulting from mutations in the ATP-Binding Cassette, subfamily A, member 4 (ABCA4) gene. It is the most common form of inherited macular degeneration, affecting roughly in 1 in 10,000 people. The age of onset of juvenile and early adult STGD1 is usually $8-25$ years with some cases occurring in older adults (late-adult onset STGD1) [1,2]. These patients develop irreversible vision loss due to atrophy of the macular retinal pigment epithelium (RPE) and loss of photoreceptors, with patients presenting at a younger age having worse visual outcomes compared to those with later onset [1].

\subsection{Clinical Presentation}

The phenotypes of STGD1 are heterogeneous and distinct phenotypic groups of STGD1 are recognized. Early-onset childhood STGD1 typically affects children age 6 to 12 years causing rapid severe decline in visual acuity over 12 to 24 months. Clinical findings in the posterior pole are sometimes subtle, showing rare peripheral flecks and mild macular changes, but dramatic macular atrophy can be apparent on OCT and autoflouresence [3]. Juvenile and young adult forms of STGD1 have varying degree of 
macular atrophy and distribution of flecks with a wide spectrum of progression. Late onset forms of STGD1 usually have perifoveal atrophy with preserved foveal function and structure that subsequently degenerate in later adulthood [4].

A hallmark of the disease is premature accumulation of lipofuscin (a brownyellow autofluorescent pigment associated with aging) in the RPE, causing a pattern of yellowish flecks that extend outward from the macula, recently though to represent degenerated photoreceptor cells [5-7] (Figure 1). The diffuse accumulation of this lipofuscin material results in the classic dark or "silent" choroid identified on fluorescein angiography due to masking of choroidal fluorescence (Figure 2) [8]. On funduscopic examination, patients may demonstrate an atrophic or a bull's-eye appearance in the macular region, even more apparent on fundus autofluorescence (FAF) (Figure 3) $[9,10]$. Lipofuscin is also found in cells of the liver, kidney, heart muscle, adrenals, and nerves, and is considered one of the most consistent morphologic features of aging, with a degree of accumulation inversely related to age $[11,12]$. However, it is thought that the mechanisms involved in RPE lipofuscin formation are closely related to metabolic pathways that are specific to the retina and fundamentally different from mechanisms found in other tissues (described in Section 1.2) [13].

The RPE is essential for the neurosensory retina homeostasis. It acts as a transport exchange system with blood capillaries and is critical for phagocytosis of photoreceptor outer segments. However, rising RPE lipofuscin levels contributes to a decline in photoreceptor and RPE function, resulting in the degeneration of the macula with subsequent loss of central vision $[14,15]$. Figure 4 shows a spectral domain optical 
coherence tomography (SD-OCT) image of a patient with STGD1, demonstrating atrophic retina with RPE and photoreceptor irregularities.

In the Progression of Atrophy Secondary to Stargardt Disease (ProgStar) Studies, valuable information about the natural history of this disorder is being collected. Over 200 STGD1 patients were enrolled in this natural history study across multiple clinical sites. Change in best-corrected visual acuity (BCVA) over a 12-month period was minimal, and varied depending on baseline acuity, and consequently was not a sensitive 1-year outcome measure [17]. However, microperimetry is being assessed as a potentially more accurate functional outcome measure for future clinical trials [18]. In ProgStar, anatomic endpoints to assess progression of atrophy through both FAF and OCT are being assessed $[19,20]$.

\subsection{Molecular Genetics}

$A B C A 4$ acts as a membrane transporter for the recycling of chromophores (11-cisretinal) during the visual cycle. Specifically, $A B C A 4$ encodes for a photoreceptor outer segment rim protein, a "flippase" whose function is to transport the all-trans retinaldehyde-phosphatidylethanolamine (retinaldehyde-PE) Schiff base and excess 11cis-retinal from the luminal/extracellular leaflet to the cytoplasmic leaflet of rod and cone photoreceptor disc membranes, where they can then be transformed back to retinol [21, 22]. Without its functional transporter, the retinaldehyde-PE conjugates may react to form vitamin A dimers (bisretinoids, some of which include A2E and ATR-dimer), which then accumulate in the RPE after phagocytosis of the photoreceptors outer segments. Vitamin A dimers are toxic to cultured RPE cells and are thought to play a significant 
role in lipofuscin formation and subsequent retinal degeneration [23,24]. Experimental models of cultured RPE cells have suggested that A2E sensitizes RPE cells to lightinduced apoptosis [25] and has an inhibitory effect on phospholipid digestion in RPE phagolysosomes. This may result in accumulation of undigested waste products in the RPE that contributes to degeneration, though this has not been confirmed in human or STGD1 mouse models [26].

Lipofuscin also originates from the free 11-cis-retinal that is continuously supplied to the rod for rhodopsin regeneration and outer segment renewal. A2E and lipofuscin are produced in the dark as well as in the light, and $A B C A 4$ plays a role in removing 11-cis retinal in excess of that needed to combine with opsin to regenerate rhodopsin [22]. Hence, loss in the function of $A B C A 4$ can result not only in the accumulation of all-trans retinal generated by photobleaching of rhodopsin, but also excess 11-cis retinal from the visual cycle. These retinoids can then produce A2E and related compounds found in lipofuscin deposits.

More recently, the $A B C A 4$ gene was found to be expressed not only in the photoreceptor outer segments, but also the RPE cells. Consequently, $A B C A 4$ mutations may have a pathogenic role in both the photoreceptors and RPE cells, which could impact gene therapy, with regard to the need to potentially transduce both the photoreceptor and RPE layers (Farnoodian-Tedrick, et al, ARVO E-Abstract 198, 2018).

At the current time, there are no commercially available treatments for STGD1. Investigational therapies include visual cycle modulators (VCMs) that aim to reduce accumulation of Vitamin A dimers, complement inhibitors, gene therapy, and human embryonic stem-cell therapy for regeneration of the RPE. These treatments are 
summarized in Table 1.

\section{Pharmacotherapy to treat Stargardt Macular Dystrophy}

\subsection{Introduction to Visual Cycle Modulators}

The visual cycle is a series of enzymatic reactions that take place in the outer retina photoreceptors and RPE that converts all-trans retinal to 11-cis retinal for the regeneration of rhodopsin. As understanding of the visual cycle progresses, the ability to manipulate it to treat retinal disease has advanced.

The rods are single photon receptors that allow visual perception in low illumination, while the cones are less sensitive but can distinguish various wavelengths of light, to facilitate color vision. Both rods and cones use 11-cis-retinal, which binds to opsins to then form visual pigments such as rhodopsin or cone opsins [27]. When light strikes rhodopsin (composed of the protein opsin bound to the chromophore 11-cisretinal, a vitamin A derivative) in the rod outer segments, 11-cis-retinal is converted to its all-trans-retinal isomer. This, in turn, activates the opsin and initiates a signal transduction cascade, closing a cyclic GMP-gated cation channel, and hyperpolarizing the photoreceptor cell. The all-trans-retinal must be converted back to 11-cis-retinal, through a sequence of reactions catalyzed by enzymes, including retinol dehydrogenases (RDH), which catalyze reduction and oxidation reactions in the photoreceptor, as well as lecithin retinol acyltransferase (LRAT) and retinoid isomerohydrolase (a 65 kilodalton protein, encoded by the RPE65 gene), both of which are located in the RPE [28]. The visual cycle is diagrammed in Figure 5, which also shows the role of select therapeutics that influence the visual cycle. 
Although necessary for vision, 11-cis-retinal in excess (like all-trans-retinal) can be toxic due to its highly reactive aldehyde group. It must be detoxified by either reduction to retinol or sequestration within retinal-binding proteins. It has been demonstrated that $A B C A 4$ can transport N-11-cis-retinylidene- PE, the Schiff-base conjugate of 11-cis-retinal and PE, from the lumen to the cytoplasmic leaflet of disc membranes. This transport role together with chemical isomerization to its all-trans isomer and reduction to all-trans-retinol by RDH can prevent accumulation of excess 11cis-retinal and its Schiff-base conjugate, and the formation of toxic bisretinoid compounds [29].

It has been demonstrated that the RPE visual cycle, which supplies chromophore to both rods and cones, is too slow to support cone function under bright conditions [30]. Additionally, pigment regeneration is rate-limited by the supply of recycled chromophore to the photoreceptors [31], suggesting that chromophore is supplied faster to cones than to rods, possibly with the help of a second, cone-specific visual cycle. This cone-specific visual cycle depends on Müller cells instead of the RPE to regenerate chromophores [32]. This second pathway could serve as a target for future therapies, though the authors are unaware of any that exist at this time.

The visual cycle plays a key role in several retinal disorders aside from STGD1. For example, dysfunction of enzymes in the visual cycle leads to several inherited retinal diseases (IRDs) such as retinitis pigmentosa (RP) and Leber's congenital amaurosis (LCA), due to the inability to either produce an adequate supply of 11-cis-retinal or an inability to remove the accumulation of various retinoid products. The visual cycle has become the focus of therapeutic strategies as several compounds have the potential to 
address defects in this cycle to treat rare IRDs. Several clinical trials have assessed these investigational VCMs with the goal of potentially slowing the progression of STGD1 and age-related macular degeneration (AMD), the leading cause of irreversible blindness in the industrialized world.

\subsection{Decrease Toxic Byproducts of $A B C A 4$ Dysfunction: ALK-001 (C20-D3-vitamin} A)

The rate determining step in vitamin A dimerization is the cleavage of a C20 carbon-hydrogen bond of the retinaldehyde-PE Schiff base [33]. Replacing the C20 hydrogen atoms of vitamin A with deuterium atoms (i.e. C20-D3 -vitamin A) makes this bond harder to cleave and impedes vitamin A dimerization. Several studies have sought to determine whether slowing the intrinsic reactivity of vitamin A to dimerize could slow lipofuscin formation in the RPE and delay changes associated with human STGD1. In an experimental mouse model of STGD1, $A B C A 4^{-/-}$mutant albino mice were raised on diets containing either C20-D3 -vitamin A (the treated group) or vitamin A at its natural isotopic abundance (the control group). The concentration of vitamin A dimers, lipofuscin and other biological markers indicative of ocular health in both groups were measured. Treated mice exhibited an $80 \%$ reduction in A2E, a 95\% reduction in ATR dimer and a $70 \%$ decrease in fundus autofluorescence at three months of age. After six months, the treated group showed fewer lipofuscin granules as visualized qualitatively by electron microscopy, and at 12 months they showed improved eye function as measured by electroretinogram (ERG). These results suggest that pathological phenotypes that arise from defects in the $A B C A 4$ gene may result from the dimerization of vitamin A and may 
be improved by hindering the ability of vitamin A to dimerize [34].

Similar results were found in another mouse model of STGD1, in which Vitamin A dimerization contributed to over $50 \%$ of lipofuscin accumulation and caused transcriptional dysregulation of several complement genes associated with inflammation [35]. Replacing Vitamin A with C20-D3-vitamin A impeded dimerization of Vitamin A (by approximately five-fold for A2E), and additionally normalized the aberrant transcription of complement genes without impairing retinal function. Phenotypic rescue by C20-D3-vitamin A was also observed noninvasively by quantitative autofluorescence in as little as 3 months after the initiation of treatment, whereas upon interruption of treatment, the age-related increase in autofluorescence resumed. These results further indicate that administration of C20-D3 -vitamin A may be a feasible therapeutic approach to slow the progression of associated retinal disease caused by Vitamin A dimerization. During these mice studies, no side effects were noted, and the animals were administered the drug for 12 months.

The promising results of the aforementioned pre-clinical studies have paved the way for the oral once-daily C20-D3-vitamin A molecule, ALK-001 (Alkeus Pharmaceuticals, Boston, MA), to begin human clinical trials in STGD1. A Phase 1 trial (NCT02230228) to assess the safety and pharmacokinetics in healthy volunteers has been completed [36]. The phase 2 TEASE study (NCT02402660) is ongoing [37].

\subsection{Decrease Toxic Byproducts of $A B C A 4$ Dysfunction: VM200}

Vision Medicine's VM200 molecule for STGD1 is currently in pre-clinical trials. This oral aldehyde trap sequesters the toxic compound, all-trans retinal, to potentially 
prevent retinal cell death [38]. Specifically, VM200 is a primary amine that reacts with the aldehyde group of all-trans retinal to form an inactive Schiff base, thus making it unable to form A2E. VM200 was shown to preserve retinal structure in $A B C A 4^{-/-} \mathrm{Rdh}^{-/-}$ mice, as measured by SD-OCT. According to unpublished data from Case Western Reserve University, VM200 has also demonstrated ability to preserve retinal function, as mice treated with it were noted to have increased concentration of 11-cis retinal (a biomarker of intact photoreceptors) compared to controls [39]. No significant toxicities were noted in 2-week and 13-week long studies. The molecule of VM200 is an enantiomer of pregabalin, which is used to treat neuropathic pain, though its affinity for the pregabalin target is 10 -fold less than that of pregabalin. VM200 could also have therapeutic potential in other inborn errors of aldehyde metabolism including SjogrenLarsson Syndrome, Best Disease, and Succinic semialdehyde-dehydrogenase deficiency. Pre-clinical studies are continuing [39].

\subsection{Inhibition of 11-cis-retinol dehydrogenase: Isotretinoin}

Isotretinoin (Accutane) is a drug indicated for the treatment of acne. It has also been shown to inhibit lipofuscin formation in a mouse model [40] by inhibiting 11-cisretinol dehydrogenase in the visual cycle, thus slowing the synthesis of 11-cisretinaldehyde and regeneration of rhodopsin. This explains the side effect of decreased night vision in patients who use isotretinoin for acne [41], though isotretinoin has not been shown to induce photoreceptor degeneration, and actually protects against lightinduced damage [42]. Light activation of rhodopsin results in its release of all-transretinaldehyde, which constitutes the first reactant in toxic $\mathrm{A} 2 \mathrm{E}$ biosynthesis. $A B C A 4^{-/-}$ 
mice that were injected with isotretinoin had decreased production of A2E, along with less formation of lipofuscin granules in the retina compared to controls, as viewed by electron microscopy. Additionally, wildtype mice treated with isotretinoin for 2 months had a $40 \%$ reduction of A2E formation in the RPE compared to controls. On ERG, both wild-type and $A B C A 4^{-/-}$mice showed smaller delays in dark adaptation after isotretinoin administration with bright compared with dim probe flashes. These results suggest that isotretinoin reduced rhodopsin levels in both wild-type and $A B C A 4^{-/ /}$retinas. The authors propose that isotretinoin may delay visual loss in STGD1 and other retinal diseases linked to lipofuscin accumulation.

\subsection{Inhibition of RPE65: Emixustat.}

Emixustat (ACU-4429, developed by Acucela Inc) is a small non-retinoid derivative of retinylamine that inhibits retinoid isomerohydrolase (encoded by the RPE65 gene), thus reducing the conversion of all-trans-retinyl ester to 11-cis-retinol and preventing accumulation of A2E. Phase 1 studies showed that the drug was well-tolerated up to $75 \mathrm{mg}$ with expected dose dependent suppression of scotopic ERG in healthy subjects [43]. It was initially developed as an investigational agent to potentially slow the progression of geographic atrophy (GA) in age-related macular degeneration (AMD), but it is also being assessed as a potential treatment for STGD1.

In May 2016, Acucela announced the results of its Phase 2b/3 "S.E.A.T.T.L.E." clinical trial, which was designed to determine if emixustat could reduce the growth rate of GA compared to placebo. The study failed to meet its primary endpoint, as there was no statistically significant difference in lesion growth rate for any treatment group 
compared to placebo. There was no significant difference in the mean change of BCVA from baseline to month 24 between treatment groups. There was a small numerical treatment difference observed in certain patients with specific genetic profiles in favor of emixustat. The profile of adverse events was similar to that of earlier trials [44]. Acucela is currently assessing emixustat as a potential treatment for STGD1, and approximately 30 patients were enrolled in a phase 2a study in the United States [45], and phase 2b/3 study is planned.

\subsection{Retinol-binding protein antagonists: Fenretinide}

Fenretinide (Sirion Therapeutics) is an oral synthetic retinoid derivative that competes with retinol to bind with retinol-binding protein 4 (RBP4), thus preventing transport of retinol into the RPE. Serum retinol is maintained in circulation as a tertiary complex with RBP4 and transthyretin (TTR). Reduction in delivery of retinol-RBP-TTR to the RPE is thought to decrease accumulation of A2E, and may potentially slow vision loss in patients with STGD1 and AMD. Once fenretinide binds to RBP, the RBPfenretinide complex is rapidly eliminated in the urine [46]. Fenretinide has been shown to reduce formation of A2E in a mouse model of STGD1 [47]. Possible downsides to fenretinide therapy include its tendency to induce apoptosis in many cell types (including RPE) [48], along with teratogenic effects that would limits its use in women of childbearing age (more pertinent in treating STGD1 than AMD) [49].

A recent phase 2 study assessed fenretidine (100 and $300 \mathrm{mg}$ orally administered daily versus placebo) for slowing lesion growth in 246 patients with GA [50]. There was a dose-dependent reduction of serum RBP in fenretinide-treated patients. There was also 
a trend for reduced annual lesion growth rates in patients in the $300 \mathrm{mg}$ fenretinide group who achieved serum retinol levels of $\leq 1 \mu \mathrm{M}\left(1.70 \mathrm{~mm}^{2} /\right.$ year vs. $2.03 \mathrm{~mm}^{2} /$ year, mean reduction of $0.33 \mathrm{~mm}^{2}$ compared to placebo, $\mathrm{p}=0.1848$ ). Only $51 \%$ of patients receiving $300 \mathrm{mg}$ and completing the 2-year study achieved this level of serum retinol reduction, resulting in a non-significant change in lesion growth rate versus the placebo group. RBP reductions $<2 \mathrm{mg} / \mathrm{dL}$ correlated with further reductions in lesion growth rates $\left(\mathrm{r}^{2}=\right.$ 0.478). There was a $45 \%$ reduction in $\mathrm{CNV}$ formation among fenretinide groups, though all groups in the study lost a mean of 10-11 letters of vision at 2 year follow up, consistent with the natural history of GA and suggesting no visual benefit to the modest reduction in GA growth [50]. Furthermore, in the $300 \mathrm{mg}$ fenretinide group, $20.2 \%$ of patients withdraw from the study due to adverse effects, though only complaints related to the skin or eye were thought to be drug related. The most common ocular adverse events that were reported (but did not necessarily lead to study withdrawal) included decreased visual acuity (71 \%), night blindness (37.3\%), and visual disturbance (26.5 $\%)$.

\subsection{Retinol-binding protein antagonists: A1120}

A1120 (ICR-14967) is another drug that lowers serum retinol levels as a mechanism of potential treatment of STGD1. It was originally developed as a potential treatment for diabetes. Like fenretinide, A1120 is a RBP4 antagonist, though A1120 differs in that it is not a retinoid and not an agonist to Retinoic Acid Receptor-alpha. This property may spare patients from the side effect profile associated with retinoids, which includes nyctalopia and delayed dark adaptation. $A B C A 4^{-/}$mice that were administered 
A1 $12030 \mathrm{mg} / \mathrm{kg}$ daily for 6 weeks were found to have a 75\% reduction of serum RBP4 along with 50\% reduction of lipofuscin bisretinoids compared to the control group. On ERG testing, no statistically significant difference in kinetics of the b-wave recovery after photobleaching was found between the groups of A1120- and vehicle-treated wild-type animals, suggesting that A1120's capacity for reduction of lipofuscin bisretinoids in the retina may not be associated with the appreciable suppression of the visual cycle. This finding supports the notion that A1 120 is unlikely to cause side effects of nyctalopia or delayed dark-adaptation, which is seen in the other visual cycle modulators [51]. Based on this preclinical data, clinical trials are under development by a collaborative effort between iCura Vision, Columbia University, and the National Institutes of Health [51].

\subsection{Complement C5 inhibition: avacincaptad pegol (Zimura $\left.{ }^{\circledR}\right)$}

A2E and other bisretinoids are thought to have a pro-inflammatory effect by activation of the complement system in RPE cells [52-54]. Increased complement activation and inflammatory markers have been observed in $A B C A 4^{-/-}$mice, which has been associated with RPE dysfunction and photoreceptor degeneration [55]. C5 complement plays a role in the terminal portion of the complement pathway, where the lectin, classical, and alternative complement pathways merge to form an inflammasome (a multi-protein complex responsible for activation of inflammatory responses) and membrane attack complex (MAC, involving complement $\mathrm{C} 5 \mathrm{~b}-9$ ), both of which result in cell death [56].

The antioxidant resveratrol and anti-C5 antibody have been shown to protect against RPE cell death in a cell culture model in which the RPE cells were exposed to 
bisretinoid-induced alternate complement pathway activation [54]. In a model of $A B C A 4^{-}$

I- mice, viral vector gene therapy was used to increase expression of complement receptor 1-like protein (CRRY), a complement negative regulatory protein, in the RPE. This resulted in reduced complement levels of $\mathrm{C} 3 / \mathrm{C} 3 \mathrm{~b}$ in the $\mathrm{RPE}$, along with a 2-fold reduction in bisretinoid accumulation compared to sham-injected $A B C A 4^{-/-}$mice. On histologic evaluation, there were $30 \%$ fewer lipofuscin granules and significantly more photoreceptor nuclei in the outer nuclear layer compared to sham-injected $A B C A 4^{-/-}$mice after one year [57]. This study suggested that complement inhibition could be a feasible treatment option for preventing RPE and photoreceptor degeneration in STGD1.

Avacincaptad pegol (Zimura ${ }^{\circledR}$, Ophthotech Corporation) is a C5 complement inhibitor which may prevent formation of the MAC, and consequently reduce cell death caused by destruction of the cell membrane. It is delivered by intravitreal injection and is being explored as a potential treatment option for a number of eye diseases including STGD1, dry AMD (GA), wet AMD, idiopathic polypoidal choroidal vasculopathy, and posterior uveitis [58]. A phase 2b study (NCT03364153) was initiated at the beginning of 2018, which plans to follow approximately 120 STGD1 patients for 18 months. The primary endpoint of the study will assess the mean rate of change of the area of ellipsoid zone defects as measured by SD-OCT (Csaky KG, et al, ARVO E-Abstract 1569, 2018). For Dry AMD (GA) patients, a phase 1/2a clinical trial has already been completed, which showed that intravitreal injections of avacincaptad pegol were well-tolerated and there were preliminary signs of a relative dose-dependent decrease in growth of GA after monthly injection for 36 weeks $[58,59]$.

\section{ABCA4 Gene Therapy for Stargardt Macular Dystrophy}


The goal of gene therapy for STGD1 is to introduce a functional $A B C A 4$ gene to the retina, which will allow for expression of the membrane transporter and reduce buildup of toxic vitamin A dimers in the RPE.

\subsection{Introduction to viral vectors for gene therapy}

A variety of viral and non-viral gene delivery methods have been developed over the past two decades. Choosing one delivery method over another is influenced by the tissue to be targeted, the cloning capacity of the vector (which determines the size of the expression cassette that can be accommodated in the genome of the virus), longevity of expression, and safety concerns (inflammatory responses, and possibility of genotoxicity/insertional oncogenesis). Adeno-associated virus (AAV) and lentivirus vectors have been used in the treatment of inherited retinal diseases, with AAV being the most common viral vector utilized [60].

\subsection{Methods of introducing viral vectors to the eye}

Due to the ability to monitor functional changes in retinal disease, the eye provides an excellent model for investigating gene therapy. Additionally, the relative ocular immune-privilege limits an immune response to the implanted genetic material. Additional advantages include ease of accessibility for delivery of the genetic material directly to the target cells of interest, and the non-invasive ability to monitor for disease progression and response to therapy [61].

Vector delivery to the target retinal tissue involves two potential methods. The most commonly investigated method involves pars plana vitrectomy (PPV) followed by retinotomy and injection of the viral vector with genetic material into the subretinal space. This more invasive method creates a temporary retinal detachment, but allows for 
direct delivery to the cells of interest. The virus then "infects" the RPE cells or photoreceptors, causing the host cells own translational machinery to express the protein. Alternatively, injection of the vector into the vitreous cavity has been attempted, and although this method may be less invasive and potentially has fewer procedure-related complications, the penetration of viral vector to the target tissue is perceived to be inferior to that of subretinal injections [62]. Additionally, animal models have

demonstrated an induced humoral immune response to intravitreally delivered vectors that was not observed with subretinally delivered vectors [63].

\subsection{EIAV-ABCA4 for Stargardt Macular Dystrophy}

The $A B C A 4$ gene (6.8 kb) far exceeds the $4.5-5.0 \mathrm{~kb}$ capacity of the AAV vector, leading to utilization of an equine infectious anemia lentivirus (EIAV) for gene transfer [64]. Subretinal injection of EIAV-ABCA4 was found to be effective in a knock out $A B C A 4^{-/-}$mouse model, in which treated eyes had a significant reduction of A2E concentrations compared to untreated and mock-treated (EIAV-null vector) control eyes. Treated eyes of $A B C A 4^{-/-}$mice accumulated 8-12 pmol per eye (s.d.=2.7) of A2E 1 year after treatment, amounts similar to wild-type controls, whereas mock-treated or untreated eyes had 3-5 times more A2E (27-39 pmol per eye, s.d.=1.5; $\mathrm{P}=0.001-0.005)$ [44].

Further animal studies tested the safety and distribution of EIAV-ABCA4 in rabbits and macaques. In both species, there was a slightly higher amount of intraocular inflammation in eyes receiving the subretinal injection, though it was localized, transient, and did not cause toxicity. Intraocular pressure and ERG were unaffected, while histopathological evaluation did not reveal any detrimental effects of the subretinal 
injection. The vector remained in the ocular compartment, though a serum antibody response was observed in rabbits but not macaques, and did not result in any long-term systemic toxicity [65].

Currently, Oxford Biomedica in coordination with Sanofi, is sponsoring an escalating dose Phase I/II clinical trial of SAR422459, formerly known as StarGen ${ }^{\text {TM }}$ (NCT 01367444), investigating safety and preliminary signs of efficacy in STGD1 over a 48 week follow up period [66] [60]. The trial, which plans to enroll an estimated 46 patients, began in June 2011 and has an expected completion date in November 2019. Another study (NCT01736592) is assessing the long-term safety and tolerability of patients in the original SAR422459 trial, in which these patients are followed without further intervention for 15 years.

Another approach to address the large size of the $A B C A 4$ gene, employs a dual AAV strategy instead of Lentivirus vector, which splits the gene into two AAV vectors. Inside the host cell, the two transgene fragments combine to generate the $A B C A 4$ gene. One group recently presented preclinical work in a $A B C A 4$ knock out mouse model showing that dual AAV vectors can be used to deliver $A B C A 4$ gene, express protein, and have an effect on lipofuscin/A2E accumulation in this model (Dyka FM, et al. ARVO EAbstract 4533, 2018; Garanto A, et al. ARVO E-Abstract 4532, 2018).

An alternative to viral vector gene therapy is nanoparticle technology, which can accommodate large genes such as $A B C A 4$ for injection into the subretinal space, have historically not shown long term gene expression in animal models. However, in a recent STGD1 mouse model, eyes that were injected with subretinal $A B C A 4$ nanoparticles were found to have $A B C A 4$ transgene expression that peaked at 2 months and lasted for up to 8 
months. The eyes showed reduced lipofuscin granules on histopathologic evaluation, and improvement of dark-adaptation recovery on ERG [67]. Based on these results, further study with nanoparticle gene therapy is warranted.

Another strategy involves oligonucleotides to address common splice defects in the $A B C A 4$ gene. At least two groups have presented preclinical work supporting this route for these specific mutations (Dulla K, et al. ARVO E-Abstract 5315, 2018). Antisense oligonucleotide-based treatment restores $A B C A 4$ splicing defects caused by deep-intronic mutations associated with STGD1. Given the effect is not long lasting, repeat treatments via intravitreal injections have been proposed.

\section{Stem Cell Therapy for Stargardt Macular Dystrophy}

\subsection{Introduction to stem cell therapy for retinal disease}

Given that RPE cell dysfunction is thought to play a major role in the pathogenesis of STGD1, stem cell therapy has been explored as an option to rejuvenate or replace these damaged RPE cells. Stem cells can be derived from human embryonic stem cells (hESCs), induced pluripotent stem cells (iPSCs), and adult stem cells. Human ESCs have a great ability to divide and differentiate into a variety of cell types [68]. Induced pluripotent stem cells (iPSCs) are intriguing in that they can be derived from a previously differentiated cell source and have the potential for reduced ethical controversy over hESC- based therapies, and may negate immunological issues associated with hESC-based therapies. Like with gene therapy, the eye is an ideal candidate for stem cell research because the clear ocular media allows for direct visualization of transplanted cells and the eye is a relatively immune privileged site; 
furthermore, the size of the eye requires smaller quantities of therapeutic tissue in comparison to other organs [69]. Preclinical studies of hESC-derived RPE cells transplanted into the subretinal space of mouse models of retinal degeneration have been well tolerated and the cells sustained visual function and photoreceptor integrity in a dose-dependent fashion [70, 71].

\section{2 hESC-derived RPE cell via subretinal transplantation for STGD1}

Given the success of preclinical studies, phase I/II clinical trials were developed to test safety and preliminary signs of efficacy of subretinal transplantation of hESCderived RPE cells in patients with STGD1 and AMD (NCT01345006/NCT01469832). Nine subjects with atrophic AMD and nine with STGD1 were included and all had baseline visual acuity measuring from 20/200 to hand motion [72]. Patients underwent pars plana vitrectomy, followed by injection of $150 \mu \mathrm{l}$ of terminally differentiated RPE cellular suspension into the subretinal space at preselected sites of transition between diseased and healthy retina. Patients received either 50,000 cells, 100,000 cells, or 150,000 cells and were immunosuppressed with tacrolimus and mycophenolate mofetil from 1 week before surgery until 12 weeks following surgery, and thereafter as determined on individual basis [72].

Following surgery, no signs of acute rejection were noted, such as prominent lymphocyte infiltration, acute or chronic moderate grade noninfectious uveitis, hyperacute rejection, cystoid macular edema, persistent retinal detachment, encapsulation of the transplanted cells, or whitening of the transplanted area. No eyes developed abnormal growth suggestive of a teratoma, a tumor comprised of two or more germ 
layers, which was seen in animals transplanted with suspension of RPE cells spiked with these hESC. No eyes developed proliferative vitreoretinopathy or a retinal detachment, although four patients developed a cataract and one developed endophthalmitis not associated with a subretinal abscess. These results support safety and tolerability of subretinal stem cell-derived RPE therapy [72].

Although visual outcomes without randomization and control group in phase $1 / 2$ trials are inconclusive, no alarming functional adverse events were noted. No significant changes were seen in visual field, static perimetry, ERG, or reading speed. Treated eyes that did not develop cataracts during at least 6 months of follow-up ( $\mathrm{n}=5)$ improved by a median of ten letters at 1 month, 15 letters at 6 months, and 12 letters at 12 months whereas the fellow untreated eyes improved by a median of four letters at 1 month, four letters at 6 months, and two letters at 12 months. The National Eye Institute Visual Function Questionnaire revealed an improvement in the mental health and vision subscales for general vision, peripheral vision, near activities, and distance activities by a median of 8-20 points 3-12 months after transplantation of hESC-RPE [72]. A long-term follow-up study (NCT02445612) is following the same cohort of patients up to five years after stem cell implantation. Three-year results supported continued safety of the hESCRPE transplantation. The STGD1 patients gained a mean of 4.1 ETDRS letters in the treated eye and 3.3 letters in the untreated eye at 3 year follow up (5.8 and 4.0 letters, respectively, when excluding visually significant cataract) (Schwartz SD, et al. ARVO EAbstract 5004, 2018). Further studies by the same company will continue to explore safety and potential efficacy of hESC-derived RPE cells utilizing an updated cell line compliant with the current guidelines. 


\section{Conclusion}

While there are currently no commercially available treatments for STGD1, there are several categories of therapeutics under investigation to potentially fulfill this unmet need. These include investigational VCMs, complement inhibitors, subretinal $A B C A 4$ gene replacement therapy (with viral vectors or nanoparticles), and subretinal transplantation of stem cell-derived RPE cells.

Pharmacological modulation of the visual cycle serves as a novel approach to the potential treatment of degenerative retinal diseases. Four classes of therapeutics, with mechanisms of action involving inhibition of vitamin A dimer accumulation in the retina, are emerging as potential treatments for STGD1. These include direct inhibitors of key visual cycle enzymes (isotretinoin and emixustat), RBP4 antagonists (fenretinide and A1120), primary amine-containing aldehyde traps (VM200), and deuterated analogs of vitamin A (ALK-001). Avacincaptad pegol is an investigational C5 complement inhibitor that aims to reduce inflammation-induced RPE cell death.

Gene therapy with subretinal injection of EIAV-ABCA4 (SAR422459) has been well-tolerated and successful in reducing A2E accumulation in animal models, thus paving the way for a phase I/II clinical trial that is expected to complete at the end of 2019. A Phase I/II trial of subretinal transplantion of hESC-derived RPE cells has demonstrated safety and tolerability in a small cohort of STGD1 patients.

\section{Expert Opinion}

As the pathophysiology of STGD1 is complex and not fully comprehended, a 
multi-targeted approach may be necessary to maximally halt the disease processes. Lipofuscin/bisretinoid accumulation, activation of the complement system, and chronic inflammation are all intertwined processes that could contribute to STGD1 progression to varying degrees. The emixustat and fenretinide trials both failed to show a statistically significant improvement in GA progression or BCVA in AMD patients, and also caused a notable amount of ocular and non-ocular side effects. Still, their manufacturers hope for better results with these two drugs to prevent vision loss in STGD1 patients.

Since success in mouse models has not translated to meaningful success in human subjects, it is unclear if A2E reduction will enhance visual function in humans. There are many issues that can plague a clinical trial - for instance in a mouse model the phenotype is fairly constant. This is often not the case in human disease. The selection of patients in terms of disease stage is a difficult matter especially in clinical trials evaluating safety. Additionally, it is doubtful that patients in the real world would show compliance with a drug that causes nyctalopia and impaired dark adaptation. These are some of the issues that must be resolved before investigational VCMs become a feasible treatment option for STGD1.

Isotretinoin, which has been used as an acne treatment for many years with an acceptable safety proposal (aside from teratogenicity), has shown some promise in reducing lipofuscin formation in mouse models of STGD1, but there is a paucity of data to confirm its beneficial effect on humans with STGD1 at this point in time. C20-D3vitamin A (ALK-001) has shown similarly impressive results in reducing accumulation of Vitamin A dimers in the retina, along with improved ERG function in Stargardt mouse models, though the results of the completed phase 1 trial have not been released, to the 
best of the authors' knowledge.

For management of STGD1, A1120, a RBP4 antagonist that lowers serum retinal levels, may one day serve as an intriguing option, as its developer suggests that it may not be associated with mechanism-based ocular side effects typical for direct visual cycle inhibitors, such as nyctalopia and delayed dark-adaptation [36]. However, given that it has only been tested in mouse models of STGD1, it is too soon to speculate about its potential efficacy in humans. Aldehyde trapping (VM200) could also theoretically represent an appealing approach to inhibiting retinal bisretinoid formation, as it may also lack the mechanism-based ocular side effects typical for direct visual cycle inhibitors. However, these compounds may need to be administered at very high systemic doses to act as aldehyde traps in the retina, which raises safety concerns. Local retinal delivery may be considered as an alternative to systemic administration of aldehyde traps to overcome potential systemic toxicities [36].

Complement inhibition has been investigated as a treatment to potentially prevent progression of GA in dry AMD, but results have not been encouraging thus far, which casts uncertainty about potential efficacy for STGD1. Despite promising phase 2 results, intravitreal injections of the alternative pathway complement factor D inhibitor, lampalizumab (Roche/Genentech) failed to reduce GA area change from baseline in the twin phase 3 CHROMA and SPECTRI studies [37]. Intravenous infusion of the C5 complement inhibitor, eculizumab (Alexion pharmaceuticals) also failed to significantly decrease the growth rate of GA in the phase 2 COMPLETE study [39]. It is unclear if avacincaptad pegol, another C5 complement inhibitor, will be more successful in AMD/STGD1, but perhaps intravitreal injection delivery may allow for better retinal 
drug levels than intravenous infusion.

Viral vector gene therapy has been approved for RPE65 mutation associated retinal dystrophy $[60,73]$, which has spurred interest in gene therapy for STGD1, although the $A B C A 4$ gene is too large for AAV vectors and will require an alternative approach. Similar excitement surrounds the potential of stem cell therapy to treat STGD1. Although the Phase I/II hESC-RPE clinical trials provided encouraging safety results, the study is limited by the small sample size, relatively brief follow-up time, and lack of a control group. Further research is warranted to demonstrate safety and potential efficacy while improving methodologies of implantation.

\section{Article Highlights Box}

- Stargardt macular dystrophy (STGD1) is a hereditary retinal degeneration that may cause central vison loss and currently lacks therapies.

- Investigational oral therapies for STGD1 aim to decrease accumulation of Vitamin A dimers and lipofuscin in the retina and RPE, and include ALK-001, isotretinoin, VM200, emixustat, and A1120. There is suggestion of efficacy in mouse models of STGD1, though evidence of efficacy in humans is currently lacking.

- Visual cycle suppression is associated with nyctalopia, delayed dark-adaptation, and dyschromatopsia.

- Intravitreal avacincaptad pegol is a C5 complement inhibitor under investigation for STGD1.

- Embryonic stem cell derived RPE cell transplantation for STGD1 has shown early safety and tolerability in a small phase I/II clinical trial, Further research will 
assess potential efficacy with respect to visual function and anatomic

improvements. $A B C A 4$ gene therapy with viral vectors and nanoparticles holds

promise as a potential treatment for STGD1, though no results of a phase I/II trial

are available at this time.

\section{Bibliography}

Papers of note have been highlighted as either of interest $(*)$ or of considerable interest

$(* *)$ to readers

1. Rotenstreich Y, Fishman GA, Anderson RJ. Visual acuity loss and clinical observations in a large series of patients with Stargardt disease. Ophthalmology 2003; 110:1151-1158.

2. Fishman GA, Farber M, Patel BS, Derlacki DJ. Visual acuity loss in patients with Stargardt's macular dystrophy. Ophthalmology 1987; 94:809-814.

3. Lambertus S, van Huet RA, Bax NM, Hoefsloot LH, Cremers FP, Boon CJ, Klevering BJ, Hoyng CB. Early-onset stargardt disease: phenotypic and genotypic characteristics. Ophthalmology 2015; 122:335-344.

4. Lambertus S, Lindner M, Bax NM, Mauschitz MM, Nadal J, Schmid M, SchmitzValckenberg S, den Hollander AI, Weber BH, Holz FG, van der Wilt GJ, Fleckenstein M, et al. Progression of Late-Onset Stargardt Disease. Invest Ophthalmol Vis Sci 2016; 57:5186-5191.

5. Eagle RC, Jr., Lucier AC, Bernardino VB, Jr., Yanoff M. Retinal pigment epithelial abnormalities in fundus flavimaculatus: a light and electron microscopic study. Ophthalmology 1980; 87:1189-1200.

6. Hadden OB, Gass JD. Fundus flavimaculatus and Stargardt's disease. Am J Ophthalmol 1976; 82:527-539.

7. Paavo M, Lee W, Allikmets R, Tsang S, Sparrow JR. Photoreceptor cells as a source of fundus autofluorescence in recessive Stargardt disease. J Neurosci Res 2018

8. Uliss AE, Moore AT, Bird AC. The dark choroid in posterior retinal dystrophies. Ophthalmology 1987; 94:1423-1427.

9. Gomes NL, Greenstein VC, Carlson JN, Tsang SH, Smith RT, Carr RE, Hood DC, Chang S. A comparison of fundus autofluorescence and retinal structure in patients with Stargardt disease. Invest Ophthalmol Vis Sci 2009; 50:3953-3959.

10. Fujinami K, Lois N, Mukherjee R, McBain VA, Tsunoda K, Tsubota K, Stone EM, Fitzke FW, Bunce C, Moore AT, Webster AR, Michaelides M. A longitudinal study of Stargardt disease: quantitative assessment of fundus 
autofluorescence, progression, and genotype correlations. Invest Ophthalmol Vis Sci 2013; 54:8181-8190.

11. Brunk UT, Terman A. Lipofuscin: mechanisms of age-related accumulation and influence on cell function. Free Radic Biol Med 2002; 33:611-619.

12. Gray DA, Woulfe J. Lipofuscin and aging: a matter of toxic waste. Sci Aging Knowledge Environ 2005; 2005:re1.

13. Katz ML, Robison WG, Jr. What is lipofuscin? Defining characteristics and differentiation from other autofluorescent lysosomal storage bodies. Arch Gerontol Geriatr 2002; 34:169-184.

14. de Jong PT. Age-related macular degeneration. N Engl J Med 2006; 355:14741485.

15. Sparrow JR, Boulton M. RPE lipofuscin and its role in retinal pathobiology. Exp Eye Res 2005; 80:595-606.

16. Lee W, Noupuu K, Oll M, Duncker T, Burke T, Zernant J, Bearelly S, Tsang SH, Sparrow JR, Allikmets R. The external limiting membrane in early-onset Stargardt disease. Invest Ophthalmol Vis Sci 2014; 55:6139-6149.

17. Kong X, Strauss RW, Cideciyan AV, Michaelides M, Sahel JA, Munoz B, Ahmed M, Ervin AM, West SK, Cheetham JK, Scholl HPN, ProgStar Study G. Visual Acuity Change over 12 Months in the Prospective Progression of Atrophy Secondary to Stargardt Disease (ProgStar) Study: ProgStar Report Number 6. Ophthalmology 2017; 124:1640-1651.

18. Schonbach EM, Wolfson Y, Strauss RW, Ibrahim MA, Kong X, Munoz B, Birch DG, Cideciyan AV, Hahn GA, Nittala M, Sunness JS, Sadda SR, et al. Macular Sensitivity Measured With Microperimetry in Stargardt Disease in the Progression of Atrophy Secondary to Stargardt Disease (ProgStar) Study: Report No. 7. JAMA Ophthalmol 2017; 135:696-703.

19. Strauss RW, Munoz B, Ho A, Jha A, Michaelides M, Cideciyan AV, Audo I, Birch DG, Hariri AH, Nittala MG, Sadda S, West S, et al. Progression of Stargardt Disease as Determined by Fundus Autofluorescence in the Retrospective Progression of Stargardt Disease Study (ProgStar Report No. 9). JAMA Ophthalmol 2017; 135:1232-1241.

20. Strauss RW, Munoz B, Ho A, Jha A, Michaelides M, Mohand-Said S, Cideciyan AV, Birch D, Hariri AH, Nittala MG, Sadda S, Scholl HPN, et al. Incidence of Atrophic Lesions in Stargardt Disease in the Progression of Atrophy Secondary to Stargardt Disease (ProgStar) Study: Report No. 5. JAMA Ophthalmol 2017; 135:687-695.

21. Weng J, Mata NL, Azarian SM, Tzekov RT, Birch DG, Travis GH. Insights into the function of Rim protein in photoreceptors and etiology of Stargardt's disease from the phenotype in abcr knockout mice. Cell 1999; 98:13-23.

22. Boyer NP, Higbee D, Currin MB, Blakeley LR, Chen C, Ablonczy Z, Crouch RK, Koutalos Y. Lipofuscin and N-retinylidene-N-retinylethanolamine (A2E) accumulate in retinal pigment epithelium in absence of light exposure: their origin is 11-cis-retinal. J Biol Chem 2012; 287:22276-22286.

23. Sparrow JR, Fishkin N, Zhou J, Cai B, Jang YP, Krane S, Itagaki Y, Nakanishi K. A2E, a byproduct of the visual cycle. Vision Res 2003; 43:2983-2990. 
24. Lamb LE, Simon JD. A2E: a component of ocular lipofuscin. Photochem Photobiol 2004; 79:127-136.

25. Suter M, Reme C, Grimm C, Wenzel A, Jaattela M, Esser P, Kociok N, Leist M, Richter C. Age-related macular degeneration. The lipofusion component Nretinyl-N-retinylidene ethanolamine detaches proapoptotic proteins from mitochondria and induces apoptosis in mammalian retinal pigment epithelial cells. J Biol Chem 2000; 275:39625-39630.

26. Finnemann SC, Leung LW, Rodriguez-Boulan E. The lipofuscin component A2E selectively inhibits phagolysosomal degradation of photoreceptor phospholipid by the retinal pigment epithelium. Proc Natl Acad Sci U S A 2002; 99:3842-3847.

27. Saari JC. Vitamin A metabolism in rod and cone visual cycles. Annu Rev Nutr $2012 ; 32: 125-145$

28. Lorenz B, Gyurus P, Preising M, Bremser D, Gu S, Andrassi M, Gerth C, Gal A. Early-onset severe rod-cone dystrophy in young children with RPE65 mutations. Invest Ophthalmol Vis Sci 2000; 41:2735-2742.

29. Quazi F, Molday RS. ATP-binding cassette transporter ABCA4 and chemical isomerization protect photoreceptor cells from the toxic accumulation of excess 11-cis-retinal. Proc Natl Acad Sci U S A 2014; 111:5024-5029.

30. Mata NL, Radu RA, Clemmons RC, Travis GH. Isomerization and oxidation of vitamin a in cone-dominant retinas: a novel pathway for visual-pigment regeneration in daylight. Neuron 2002; 36:69-80.

31. Imai H, Kefalov V, Sakurai K, Chisaka O, Ueda Y, Onishi A, Morizumi T, Fu Y, Ichikawa K, Nakatani K, Honda Y, Chen J, et al. Molecular properties of rhodopsin and rod function. J Biol Chem 2007; 282:6677-6684.

32. Wang JS, Kefalov VJ. The cone-specific visual cycle. Prog Retin Eye Res 2011; 30:115-128.

33. Kaufman Y, Ma L, Washington I. Deuterium enrichment of vitamin A at the C20 position slows the formation of detrimental vitamin A dimers in wild-type rodents. J Biol Chem 2011; 286:7958-7965.

34. Ma L, Kaufman Y, Zhang J, Washington I. C20-D3-vitamin A slows lipofuscin accumulation and electrophysiological retinal degeneration in a mouse model of Stargardt disease. J Biol Chem 2011; 286:7966-7974.

35. Charbel Issa P, Barnard AR, Herrmann P, Washington I, MacLaren RE. Rescue of the Stargardt phenotype in $A B C A 4$ knockout mice through inhibition of vitamin A dimerization. Proc Natl Acad Sci U S A 2015; 112:8415-8420.

36. Petrukhin K. Pharmacological inhibition of lipofuscin accumulation in the retina as a therapeutic strategy for dry AMD treatment. Drug Discov Today Ther Strateg 2013; 10:e11-e20.

37. Twin lampalizumab trials fail to impact area of geographic atrophy. In. Ocular Surgery News.

38. Maeda A, Golczak M, Chen Y, Okano K, Kohno H, Shiose S, Ishikawa K, Harte W, Palczewska G, Maeda T, Palczewski K. Primary amines protect against retinal degeneration in mouse models of retinopathies. Nat Chem Biol 2011; 8:170-178.

39. Yehoshua Z, de Amorim Garcia Filho CA, Nunes RP, Gregori G, Penha FM, Moshfeghi AA, Zhang K, Sadda S, Feuer W, Rosenfeld PJ. Systemic complement 
inhibition with eculizumab for geographic atrophy in age-related macular degeneration: the COMPLETE study. Ophthalmology 2014; 121:693-701.

40. Radu RA, Mata NL, Nusinowitz S, Liu X, Sieving PA, Travis GH. Treatment with isotretinoin inhibits lipofuscin accumulation in a mouse model of recessive Stargardt's macular degeneration. Proc Natl Acad Sci U S A 2003; 100:47424747.

41. Weleber RG, Denman ST, Hanifin JM, Cunningham WJ. Abnormal retinal function associated with isotretinoin therapy for acne. Arch Ophthalmol 1986; 104:831-837.

42. Sieving PA, Chaudhry P, Kondo M, Provenzano M, Wu D, Carlson TJ, Bush RA, Thompson DA. Inhibition of the visual cycle in vivo by 13 -cis retinoic acid protects from light damage and provides a mechanism for night blindness in isotretinoin therapy. Proc Natl Acad Sci U S A 2001; 98:1835-1840.

43. Kubota R, Boman NL, David R, Mallikaarjun S, Patil S, Birch D. Safety and effect on rod function of ACU-4429, a novel small-molecule visual cycle modulator. Retina 2012; 32:183-188.

44. Kong J, Kim SR, Binley K, Pata I, Doi K, Mannik J, Zernant-Rajang J, Kan O, Iqball S, Naylor S, Sparrow JR, Gouras P, et al. Correction of the disease phenotype in the mouse model of Stargardt disease by lentiviral gene therapy. Gene Ther 2008; 15:1311-1320.

45. Dugel PU, Novack RL, Csaky KG, Richmond PP, Birch DG, Kubota R. Phase ii, randomized, placebo-controlled, 90-day study of emixustat hydrochloride in geographic atrophy associated with dry age-related macular degeneration. Retina 2015; 35:1173-1183.

46. Berni R, Formelli F. In vitro interaction of fenretinide with plasma retinol-binding protein and its functional consequences. FEBS Lett 1992; 308:43-45.

47. Radu RA, Han Y, Bui TV, Nusinowitz S, Bok D, Lichter J, Widder K, Travis GH, Mata NL. Reductions in serum vitamin A arrest accumulation of toxic retinal fluorophores: a potential therapy for treatment of lipofuscin-based retinal diseases. Invest Ophthalmol Vis Sci 2005; 46:4393-4401.

48. Samuel W, Kutty RK, Nagineni S, Vijayasarathy C, Chandraratna RA, Wiggert B. N-(4-hydroxyphenyl)retinamide induces apoptosis in human retinal pigment epithelial cells: retinoic acid receptors regulate apoptosis, reactive oxygen species generation, and the expression of heme oxygenase-1 and Gadd153. J Cell Physiol 2006; 209:854-865.

49. Turton JA, Willars GB, Haselden JN, Ward SJ, Steele CE, Hicks RM. Comparative teratogenicity of nine retinoids in the rat. Int J Exp Pathol 1992; 73:551-563.

50. Mata NL, Lichter JB, Vogel R, Han Y, Bui TV, Singerman LJ. Investigation of oral fenretinide for treatment of geographic atrophy in age-related macular degeneration. Retina 2013; 33:498-507.

51. Dobri N, Qin Q, Kong J, Yamamoto K, Liu Z, Moiseyev G, Ma JX, Allikmets R, Sparrow JR, Petrukhin K. A1120, a nonretinoid RBP4 antagonist, inhibits formation of cytotoxic bisretinoids in the animal model of enhanced retinal lipofuscinogenesis. Invest Ophthalmol Vis Sci 2013; 54:85-95. 
52. Zhou J, Kim SR, Westlund BS, Sparrow JR. Complement activation by bisretinoid constituents of RPE lipofuscin. Invest Ophthalmol Vis Sci 2009; 50:1392-1399.

53. Zhou J, Jang YP, Kim SR, Sparrow JR. Complement activation by photooxidation products of A2E, a lipofuscin constituent of the retinal pigment epithelium. Proc Natl Acad Sci U S A 2006; 103:16182-16187.

54. Berchuck JE, Yang P, Toimil BA, Ma Z, Baciu P, Jaffe GJ. All-trans-retinal sensitizes human RPE cells to alternative complement pathway-induced cell death. Invest Ophthalmol Vis Sci 2013; 54:2669-2677.

55. Radu RA, Hu J, Yuan Q, Welch DL, Makshanoff J, Lloyd M, McMullen S, Travis GH, Bok D. Complement system dysregulation and inflammation in the retinal pigment epithelium of a mouse model for Stargardt macular degeneration. J Biol Chem 2011; 286:18593-18601.

56. Cao S, Wang JC, Gao J, Wong M, To E, White VA, Cui JZ, Matsubara JA. CFH $\mathrm{Y} 402 \mathrm{H}$ polymorphism and the complement activation product $\mathrm{C} 5 \mathrm{a}$ : effects on NF-kappaB activation and inflammasome gene regulation. Br J Ophthalmol 2016; 100:713-718.

57. Lenis TL, Sarfare S, Jiang Z, Lloyd MB, Bok D, Radu RA. Complement modulation in the retinal pigment epithelium rescues photoreceptor degeneration in a mouse model of Stargardt disease. Proc Natl Acad Sci U S A 2017; 114:3987-3992.

58. Ophthotech Provides Update on Zimura ${ }^{\circledR}$ Complement Programs for Treatment of Eye Diseases. In.

59. Expert Insights: Will Ophthotech Continue its Zimura Development Program? In.

60. Moore NA, Morral N, Ciulla TA, Bracha P. Gene therapy for inherited retinal and optic nerve degenerations. Expert Opin Biol Ther 2017:1-13.

61. Streilein JW. Ocular immune privilege: therapeutic opportunities from an experiment of nature. Nat Rev Immunol 2003; 3:879-889.

62. Yu-Wai-Man P. Genetic manipulation for inherited neurodegenerative diseases: myth or reality? Br J Ophthalmol 2016; 100:1322-1331.

63. Li Q, Miller R, Han PY, Pang J, Dinculescu A, Chiodo V, Hauswirth WW. Intraocular route of AAV2 vector administration defines humoral immune response and therapeutic potential. Mol Vis 2008; 14:1760-1769.

64. Han Z, Conley SM, Naash MI. Gene therapy for Stargardt disease associated with ABCA4 gene. Adv Exp Med Biol 2014; 801:719-724.

65. Binley K, Widdowson P, Loader J, Kelleher M, Iqball S, Ferrige G, de Belin J, Carlucci M, Angell-Manning D, Hurst F, Ellis S, Miskin J, et al. Transduction of photoreceptors with equine infectious anemia virus lentiviral vectors: safety and biodistribution of StarGen for Stargardt disease. Invest Ophthalmol Vis Sci 2013; 54:4061-4071.

66. $\quad \mathrm{Lu}$ LJ, Liu J, Adelman RA. Novel therapeutics for Stargardt disease. Graefes Arch Clin Exp Ophthalmol 2017; 255:1057-1062.

67. Han Z, Conley SM, Makkia RS, Cooper MJ, Naash MI. DNA nanoparticlemediated $A B C A 4$ delivery rescues Stargardt dystrophy in mice. J Clin Invest 2012; 122:3221-3226. 
68. Amit M, Carpenter MK, Inokuma MS, Chiu CP, Harris CP, Waknitz MA, Itskovitz-Eldor J, Thomson JA. Clonally derived human embryonic stem cell lines maintain pluripotency and proliferative potential for prolonged periods of culture. Dev Biol 2000; 227:271-278.

69. Bracha P, Moore NA, Ciulla TA. Induced pluripotent stem cell-based therapy for age-related macular degeneration. Expert Opin Biol Ther 2017; 17:1113-1126.

70. Lund RD, Wang S, Klimanskaya I, Holmes T, Ramos-Kelsey R, Lu B, Girman S, Bischoff N, Sauve Y, Lanza R. Human embryonic stem cell-derived cells rescue visual function in dystrophic RCS rats. Cloning Stem Cells 2006; 8:189-199.

71. Lu B, Malcuit C, Wang S, Girman S, Francis P, Lemieux L, Lanza R, Lund R. Long-term safety and function of RPE from human embryonic stem cells in preclinical models of macular degeneration. Stem Cells 2009; 27:2126-2135.

72. Schwartz SD, Regillo CD, Lam BL, Eliott D, Rosenfeld PJ, Gregori NZ, Hubschman JP, Davis JL, Heilwell G, Spirn M, Maguire J, Gay R, et al. Human embryonic stem cell-derived retinal pigment epithelium in patients with agerelated macular degeneration and Stargardt's macular dystrophy: follow-up of two open-label phase 1/2 studies. Lancet 2015; 385:509-516.

73. Russell S, Bennett J, Wellman JA, Chung DC, Yu ZF, Tillman A, Wittes J, Pappas J, Elci O, McCague S, Cross D, Marshall KA, et al. Efficacy and safety of voretigene neparvovec (AAV2-hRPE65v2) in patients with RPE65-mediated inherited retinal dystrophy: a randomised, controlled, open-label, phase 3 trial. Lancet 2017; 390:849-860. 


\section{Figure Legends}

Figure 1. Fundus photo of a patient with STGD1 demonstrates atrophic retina with a "beaten bronze" appearance and surrounding yellow pisciform flecks extending outward from the fovea.

Figure 2. Fluorescein angiography demonstrates classic dark or "silent" choroid due to masking of choroidal fluorescence.

Figure 3. Fundus autofluorescence (FAF) accentuates the classic bull's eye maculopathy. Abnormally increased FAF represents excessive lipofuscin accumulation in the RPE. Inversely, decreased areas of FAF relate to low level RPE metabolic activity, which normally underlies local atrophy with secondary photoreceptor loss.

Figure 4. Spectral domain - optical coherence tomography demonstrates central retinal atrophy with outer retinal loss.

Figure 5. The visual cycle pathway begins when light (orange starburst shape) interacts with rhodopsin (green diamond shape), setting off a series of steps catalyzed by enzymes (red cloud shapes). The chemical byproducts and investigational drugs are represented in blue box and purple oval shapes, respectively. The cross-through symbol denotes an inhibitory effect of a drug on the enzymes, while a plus sign indicates the effect of increasing rhodopsin levels. $\mathrm{RDH}=$ retinol dehydrogenase, $A B C A 4=\mathrm{ATP}$-Binding Cassette Subfamily A Member 4, LRAT = lecithin retinol acyltransferase, RPE = retinal pigment epithelium. 